\title{
Undiagnosed coeliac disease at age seven: population based prospective birth cohort study
}

Polly J Bingley, Alistair J K Williams, Alastair J Norcross, D Joe Unsworth, Robert J Lock, Andrew R Ness, Richard W Jones, on behalf of the Avon Longitudinal Study of Parents and Children Study Team

Division of

Medicine,

University of

Bristol, Southmead

Hospital, Bristol

BS10 5NB

Polly J Bingley

reader

Alistair J K Williams

research associate

Alastair J Norcross

research assistant

Department of

Immunology, North

Bristol NHS Trust,

Southmead

Hospital, Bristol

BS10 5NB

D Joe Unsworth

consultant

immunologist

Robert J Lock

principal clinical

scientist

Unit of Paediatric and Perinatal

Epidemiology,

Division of Child

Deasion of Child

Health, University

of Bristol, Bristol

BS8 1TQ

Andrew R Ness

deputy director

(epidemiology),

ALSPAC

Richard W Jones

head of biological

collections, ALSPAC

Correspondence to:

PJ Bingley

polly.bingley@

bristol.ac.uk

BMJ 2004;328:322-3
Coeliac disease is uncommon in childhood and diagnosed in fewer than 1 in 2500 children in the United Kingdom. ${ }^{1}$ Subclinical disease is, however, common in adults, and can be detected by testing for serum IgA antiendomysial antibodies (IgA-EMA). ${ }^{2}$ We aimed to establish the prevalence of undiagnosed coeliac disease in the general population at age seven, and to look for associated clinical features.

\section{Participants, methods, and results}

We studied children aged 7.5 years participating in the Avon Longitudinal Study of Parents and Children (ALSPAC), a population based birth cohort study established in $1990{ }^{3}$ Two stage screening included a sensitive initial radioimmunoassay for antibodies to tissue transglutaminase (endomysial antigen) with further testing of positive samples for IgA-EMA by indirect immunofluorescence. ${ }^{4}$ Children with tTG antibodies below the 97.5th centile were defined as antibody negative. Height, weight, and haemoglobin levels were measured at dedicated study clinics. Details of gastrointestinal symptoms and special diets were collected by routine questionnaire at age 6.75 years.

Of 5470 children tested, 54 tested positive for IgAEMA $(1.0 \%$; $95 \%$ confidence interval 0.8 to 1.4$)$. IgA-EMA were more common in girls (odds ratio 2.12; 1.20 to 3.75). IgA-EMA positive children were shorter and weighed less than those who tested negative for tTG antibody ( $\mathrm{P}<0.0001$ for all comparisons). 4324 $(79 \%)$ returned questionnaires, and $50 \%$ of IgA-EMA positive children reported diarrhoea compared with $34 \%$ of tTG antibody negative (odds ratio $1.96 ; 1.06$ to 3.59). Only one IgA-EMA positive child had consulted a doctor about diarrhoea. There was no overall differ- ence in the number of episodes of diarrhoea. Vomiting, abdominal pain, and constipation were not associated with EMA, but more IgA-EMA positive children reported multiple symptoms. Only four children $(0.09 \% ; 0.1$ to 0.32$)$ were on a gluten-free diet. Of these, three were tTG antibody negative, consistent with effective treatment, and one was IgA-EMA positive (table).

\section{Comment}

At age $7,1 \%$ of children were IgA-EMA positive and likely therefore to have subclinical coeliac disease, though less than $0.1 \%$ were reported to be on a glutenfree diet. The prevalence of coeliac disease in these children is therefore comparable to that in UK adults. ${ }^{5}$ The benefit of early diagnosis of subclinical coeliac disease remains unproven, but long term follow up of this cohort may help to resolve this. If screening is worth while, it should be started in childhood.

Since ALSPAC is an observational study based on analysis of anonymous samples, ${ }^{3}$ confirmatory biopsy was not possible. IgA-EMA have however repeatedly been shown to have high sensitivity and specificity for coeliac disease, and in a recent general population study the combination of IgA-EMA and tTG antibodies that we used was associated with diagnostic histological changes in $83 \%$ of those subsequently biopsied, with abnormal intestinal $\gamma / \delta$ T-lymphocyte density in a further $12 \% .{ }^{2}$ Our strategy may even miss some affected children, as individuals with high levels of tTG antibodies without IgA-EMA may have coeliac disease. $^{2}$

Reported clinical features were similar to those in adults with coeliac disease identified by screening.

Coeliac antibody status and height, weight, haemoglobin concentration, and gastrointestinal symptoms. An additional 137 children were ITG antibody positive but IgA-EMA negative

\begin{tabular}{|c|c|c|c|}
\hline & tTG antibody negative controls & IgA-EMA positive & \\
\hline & $\begin{array}{l}\text { Median (interquartile range) } \\
\text { ( } n=5333 \text { children) }\end{array}$ & $\begin{array}{c}\text { Median (interquartile range) } \\
\text { ( } \mathrm{n}=54 \text { children) }\end{array}$ & $P$ value \\
\hline \multicolumn{4}{|l|}{$\begin{array}{l}\text { Measurements taken at age } 7.5 \\
\text { years }\end{array}$} \\
\hline Height (cm) & 126 (122.4 to 129.6) & 122.1 (118.25 to 125.33$)$ & $<0.0001$ \\
\hline Weight (kg) & 25.2 (22.8 to 28.0$)$ & 23.4 (21.35 to 25.4$)$ & $<0.0001$ \\
\hline Standard deviation score for height & $0.23(-0.43$ to 0.88$)$ & $-0.53(-1.01$ to -0.00$)$ & $<0.0001$ \\
\hline Standard deviation score for weight & $0.18(-0.45$ to 0.86$)$ & $-0.36(-1.01$ to 0.28$)$ & $<0.0001$ \\
\hline \multirow[t]{2}{*}{ Haemoglobin concentration $(\mathrm{g} / \mathrm{l})$} & 125 (120 to 130) & 123 (118 to 127) & 0.062 \\
\hline & No $(\%)$ ( $\mathrm{n}=4285$ questionnaires) & No $(\%)(n=42$ questionnaires) & Odds ratio $(95 \% \mathrm{Cl})$ \\
\hline \multicolumn{4}{|l|}{$\begin{array}{l}\text { Symptoms reported at age } 6.75 \\
\text { years }\end{array}$} \\
\hline Any diarrhoea & $1450(34)$ & $21(50)$ & 1.96 (1.06 to 3.59$)$ \\
\hline Any vomiting & $1933(45)$ & $23(55)$ & $1.47(0.80$ to 2.71$)$ \\
\hline Any stomach pains & $2557(60)$ & $28(66)$ & 1.35 (0.71 to 2.57$)$ \\
\hline Any constipation & $435(10)$ & $6(14)$ & $1.48(0.62$ to 3.52$)$ \\
\hline$\geq 3$ gastrointestinal symptoms & $931(22)$ & $17(40)$ & 2.45 (1.33 to 4.5$)$ \\
\hline
\end{tabular}


Gastrointestinal symptoms were not prominent, and the excess in girls mirrors that seen in affected adults. The most striking observation was that children with IgA-EMA were shorter by more than 0.76 standard deviation scores and lighter by 0.54 standard deviation scores than antibody negative children matched for date and place of birth. This equates to about 9 months' growth and weight gain in an average child around this age. These features were independent of gastrointestinal symptoms and anaemia and presumably unrelated to malabsorption.

Occult coeliac disease seems to start in childhood, even in those who are subsequently diagnosed as adults. The search for the trigger resulting in the breakdown of immune tolerance to gluten therefore needs to focus on infancy and intrauterine life.

We thank the children and their families taking part in the study for their continuing support and the midwives for their cooperation and help in recruitment. The ALSPAC study team comprised interviewers, computer technicians, clerical workers, research scientists, volunteers, and managers who continue to make the study possible.

Contributors: PJB, DJU, and AJKW designed the study with members of the ALSPAC study team. AJKW, RJL, and AJN developed and completed all antibody assays. RWJ coordinated the collection of biological samples and supervised handling of the samples. PJB analysed and interpreted the data with help from ARN. PJB wrote the report with help from the other authors. PJB is guarantor.

Funding: Coeliac UK, Medical Research Council, Wellcome Trust, UK government departments, and various charitable organisations and commercial companies. ALSPAC is part of the WHO initiated European Longitudinal Study of Pregnancy and Childhood.

Competing interests: None declared.

Ethical approval: ALSPAC ethics and law committee and local ethical research committees.

1 Hawkes ND, Swift GL, Smith PM, Jenkins HR. Incidence and presentation of coeliac disease in South Glamorgan. Eur J Gastroenterol Hepatol 2000; $12: 345-9$.

2 Maki M, Mustalahti K, Kokkonen J, Kulmala P, Haapalahti M, Karttunen $\mathrm{T}$, et al. Prevalence of celiac disease among children in Finland. $N$ Engl $J$ Med 2003;348:2517-24.

3 Golding J, Pembrey M, Jones R, ALSPAC study team. ALSPAC: the Avon longitudinal study of parents and children. I. Study methodology. Paediatr Perinat Epidemiol 2001;15:74-87.

4 Bazzigaluppi E, Lampasona V, Barera G, Venerando A, Bianchi C, Chiumello $\mathrm{G}$, et al Comparison of tissue transolutaminase-specific antiCho J Autoimmun 1999;12:51-6.

5 West J, Logan RF, Hill PG, Lloyd A, Lewis S, Hubbard R, et al. Seroprevalence, correlates, and characteristics of undetected coeliac disease in England. Gut 2003;52:960-5

(Accepted 11 September 2003)

\title{
Use of tobacco products as dentifrice among adolescents in India: questionnaire study
}

\author{
D N Sinha, P C Gupta, M S Pednekar
}

In India legislation prohibits the use of tobacco as an ingredient in dental care products. ${ }^{1}$ Such products, in the form of powder or paste, are applied most commonly with the index finger to teeth and gums. Various tobacco products are used as dentifrice in different parts of India. ${ }^{2}{ }^{3}$ We are not aware of any reports covering such a use of tobacco products (in contrast to chewing and smoking) especially among adolescents. ${ }^{45}$

\section{Participants, methods, and results}

The global youth tobacco survey focuses on school students aged 13-15 and uses standardised methods. ${ }^{4}$ In India the survey is being conducted independently in each state. We report on the prevalence of the use of tobacco products as dentifrice in 14 states listed in the table, with sample sizes ranging from 2067 to 5245 students.

We selected a two stage probability sample of students in grade 8-10 (which corresponds to 13-15 years in age) in each state and conducted a survey among students with an anonymous, self administered, close ended questionnaire. Participants were asked to include a maximum of five products, which could differ from state to state. A pilot tested questionnaire for India contained a specific question on application of tobacco products (in addition to chewing), and a positive response identified the use of a tobacco product as dentifrice. We defined current use as use within 30 days preceding the survey.
The overall response rate was over $80 \%$ except in Bihar (70\%) and Maharashtra (79\%). The proportion of boys was between $50 \%$ and $55 \%$ in the different states.

The use of tobacco products as dentrifice varied from $6 \%$ (Goa) to $68 \%$ (Bihar). The prevalence among boys was notably higher than among girls in Orissa and Uttaranchal, marginally higher in nine states, and marginally lower in three states. Of the specific products, tobacco toothpaste (creamy snuff) and tooth powder (lal dant manjan) were common in all states (table). Gul (a pyrolysed tobacco product) was used in eight states. Other dentifrice products containing tobacco were: mishri (roasted and powdered tobacco) and dry snuff (bajjar or tapkir) in Goa and Maharashtra; gudakhu (paste of tobacco and molasses) in Bihar, Orissa, Uttar Pradesh, and Uttaranchal; and tobacco water (tuibur or hidakphu, manufactured by passing tobacco smoke through water) in Manipur, Mizoram, Sikkim, and Tripura. It is used for gargling, not drinking.

\section{Comment}

Many students in India use tobacco products as dentifrice. Differences between the sexes were minimal and similar to those reported globally. ${ }^{5}$

In India the misconception is widespread that tobacco is good for the teeth. In a study reported from Ernakulam district in Kerala 92\% (3013) of 3261 female
School of

Preventive Oncology, A127 Anandpuri, Boring Canal Road, Patna 800001, India D N Sinha chairman

Tata Institute of Fundamental Research, Homi Bhabha Road, Colaba, Mumbai 400005 , India P C Gupta senior research scientist

Epidemiology Research Centre, Tata Memorial Centre, Ernest Borges Marg, Parel, Mumbai 400012 , India

M S Pednekar senior statistician

Correspondence to: P C Gupta pcgupta@tifr.res.in BMJ 2004;328:323-4 